\title{
Terpenes and their oxidation products in the French Landes forest: insight from Vocus PTR-TOF measurements
}

Haiyan $\mathrm{Li}^{1}$, Matthieu Riva ${ }^{2}$, Pekka Rantala ${ }^{1}$, Liine Heikkinen ${ }^{1}$, Kaspar Daellenbach ${ }^{1}$, Jordan E. Krechmer $^{3}$, Pierre-Marie Flaud ${ }^{4,5}$, Douglas Worsnop ${ }^{3}$, Markku Kulmala ${ }^{1}$, Eric Villenave ${ }^{4,5}$, Emilie Perraudin $^{4,5}$, Mikael Ehn ${ }^{1}$, Federico Bianchi ${ }^{1}$

${ }^{1}$ Institute for Atmospheric and Earth System Research / Physics, Faculty of Science, University of Helsinki, Finland

${ }^{2}$ Univ. Lyon, Université Claude Bernard Lyon 1, CNRS, IRCELYON, F-69626, Villeurbanne, France

${ }^{3}$ Aerodyne Research Inc., Billerica, Massachusetts 01821, USA

${ }^{4}$ Univ. Bordeaux, EPOC, UMR 5805, F-33405 Talence Cedex, France

${ }^{5}$ CNRS, EPOC, UMR 5805, F-33405 Talence Cedex, France

Correspondence: Haiyan Li (haiyan.li@helsinki.fi) and Matthieu Riva (matthieu.riva@ircelyon.univ-lyon1.fr) 

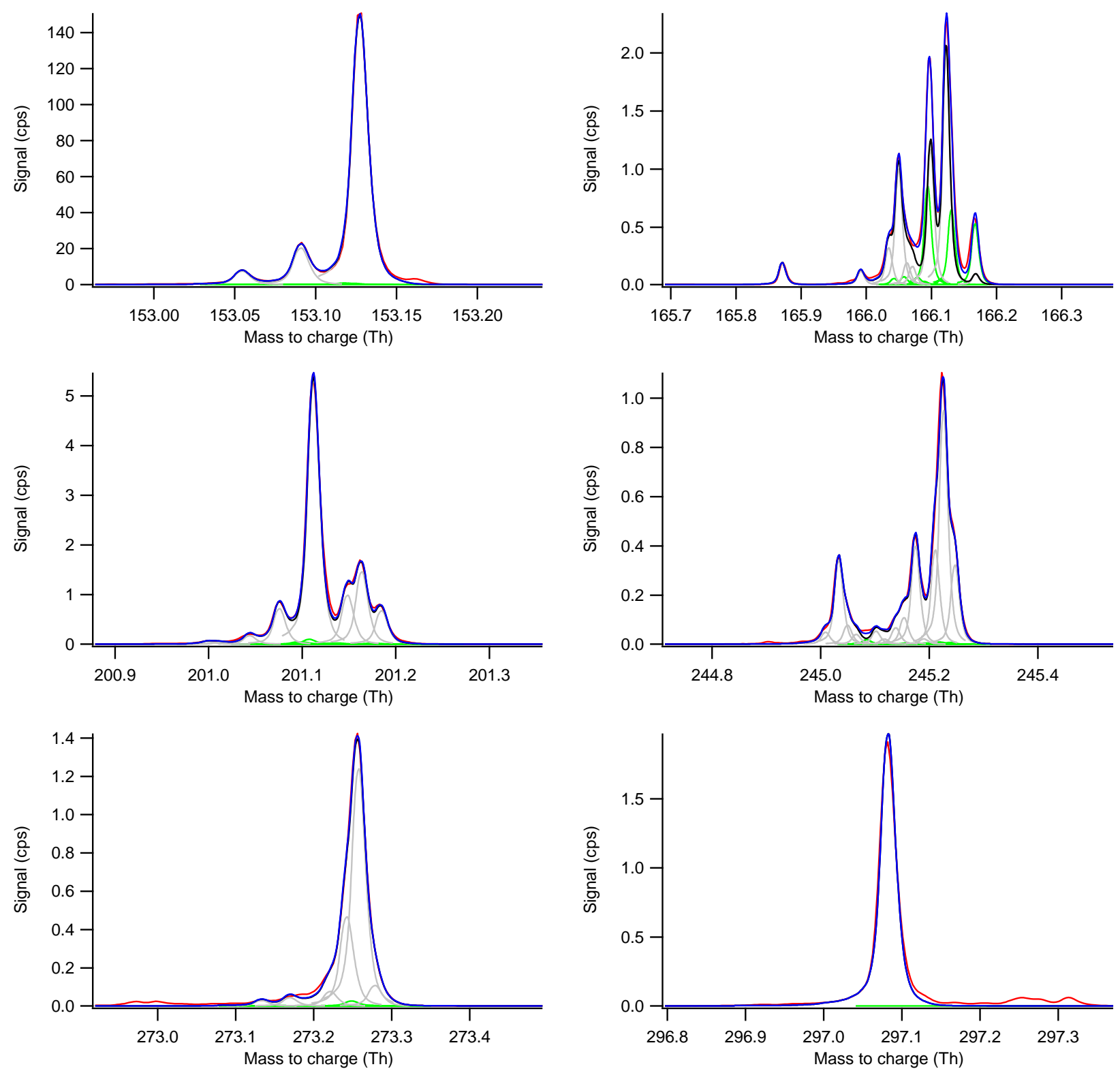

Figure S1. Examples of peak identification with the LTOF mass analyzer. 


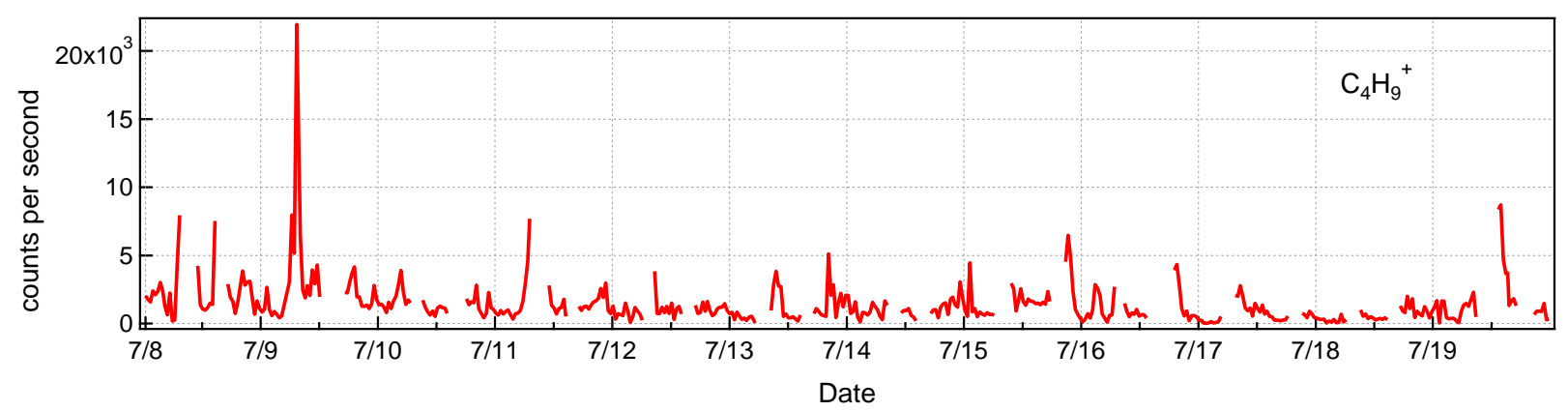

Fig. S2. Time series of the identified $\mathrm{C}_{4} \mathrm{H}_{9}{ }^{+}$. 

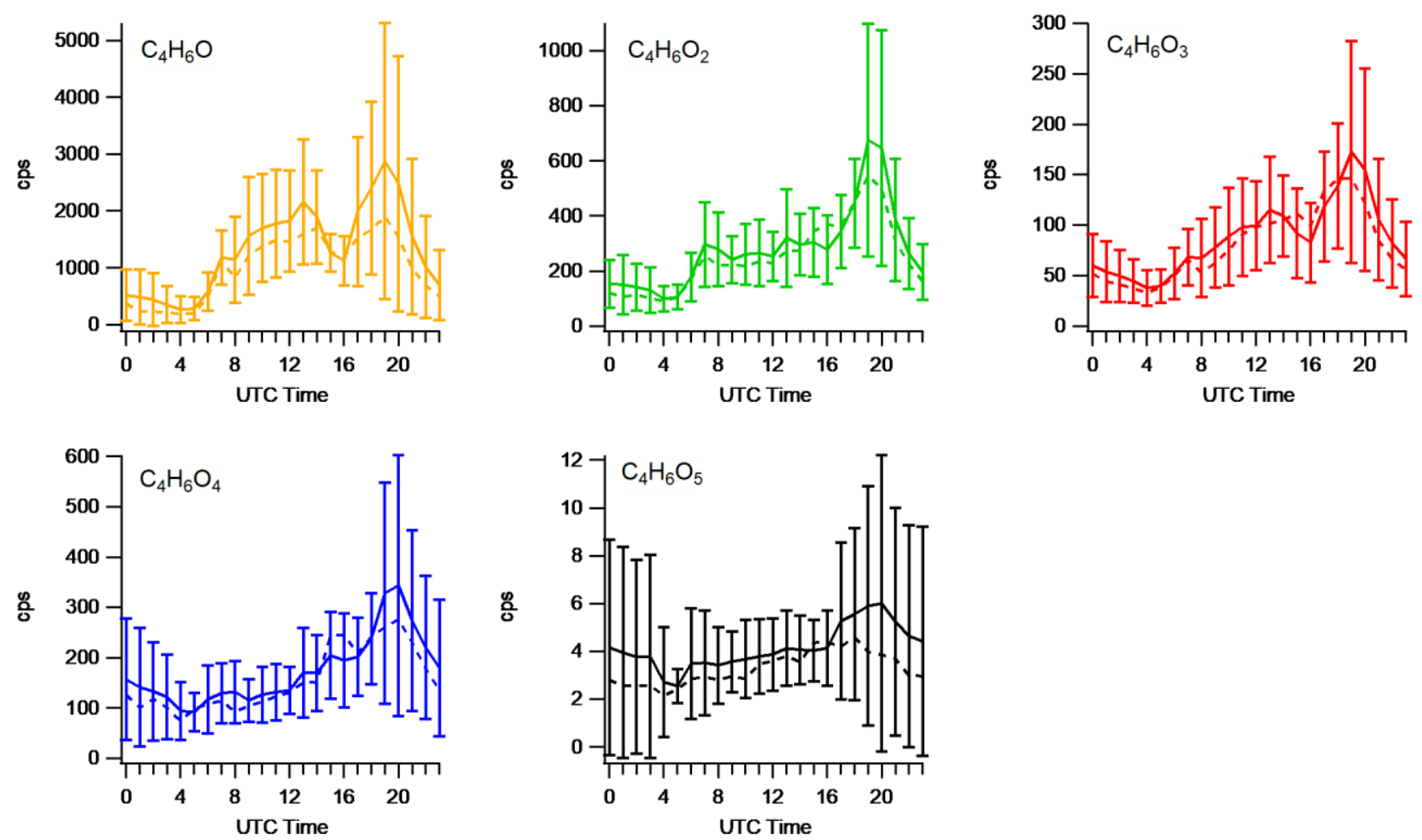

Figure S3. Diurnal patterns of non-nitrate isoprene oxidation products: (a) $\mathrm{C}_{4} \mathrm{H}_{6} \mathrm{O}$, (b) $\mathrm{C}_{4} \mathrm{H}_{6} \mathrm{O}_{2}$, (c) $\mathrm{C}_{4} \mathrm{H}_{6} \mathrm{O}_{3}$, (d) $\mathrm{C}_{4} \mathrm{H}_{6} \mathrm{O}_{4}$, and (e) $\mathrm{C}_{4} \mathrm{H}_{6} \mathrm{O}_{5}$. 

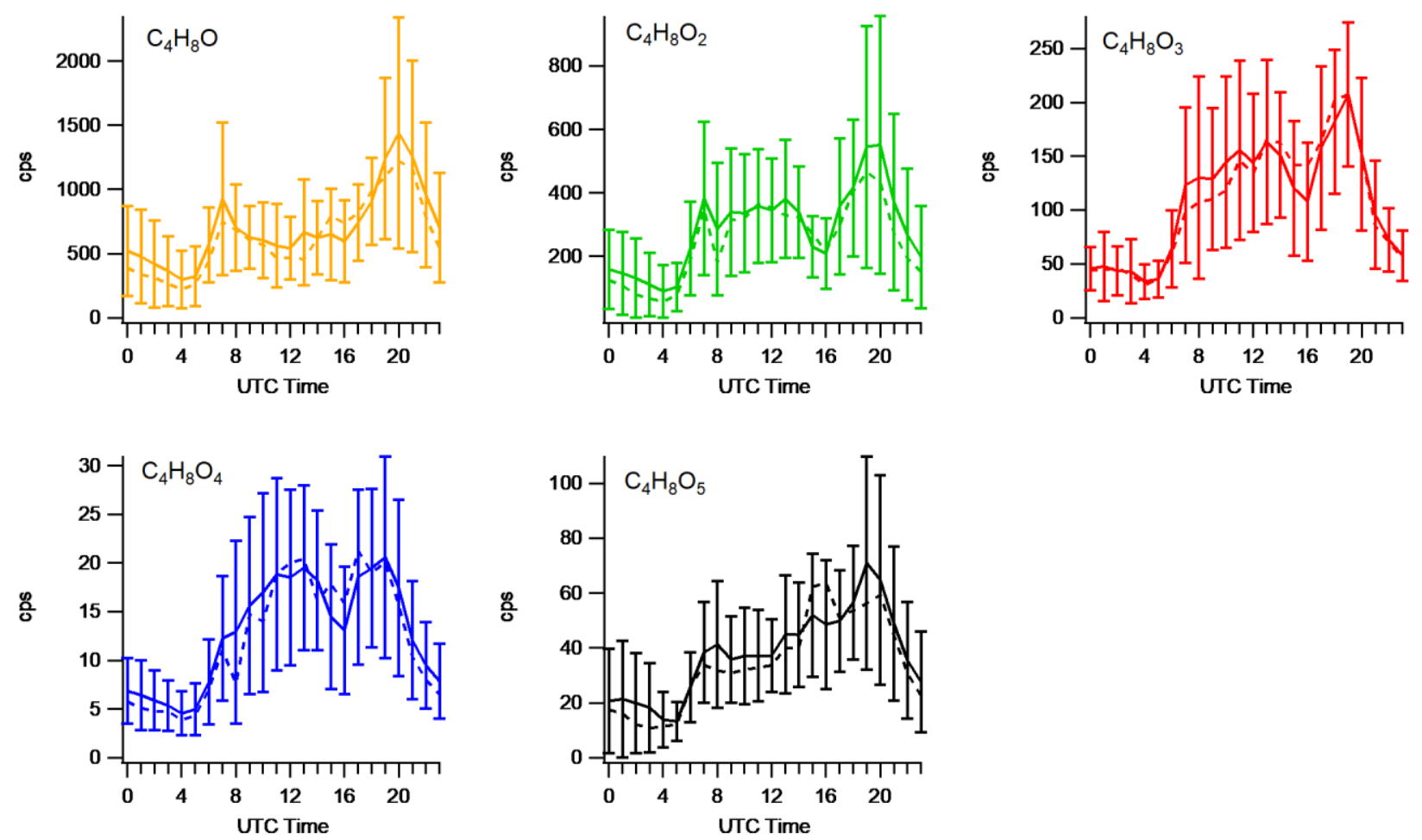

Figure S4. Diurnal patterns of non-nitrate isoprene oxidation products: (a) $\mathrm{C}_{4} \mathrm{H}_{8} \mathrm{O}$, (b) $\mathrm{C}_{4} \mathrm{H}_{8} \mathrm{O}_{2}$, (c) $\mathrm{C}_{4} \mathrm{H}_{8} \mathrm{O}_{3}$, (d) $\mathrm{C}_{4} \mathrm{H}_{8} \mathrm{O}_{4}$, and (e) $\mathrm{C}_{4} \mathrm{H}_{8} \mathrm{O}_{5}$. 

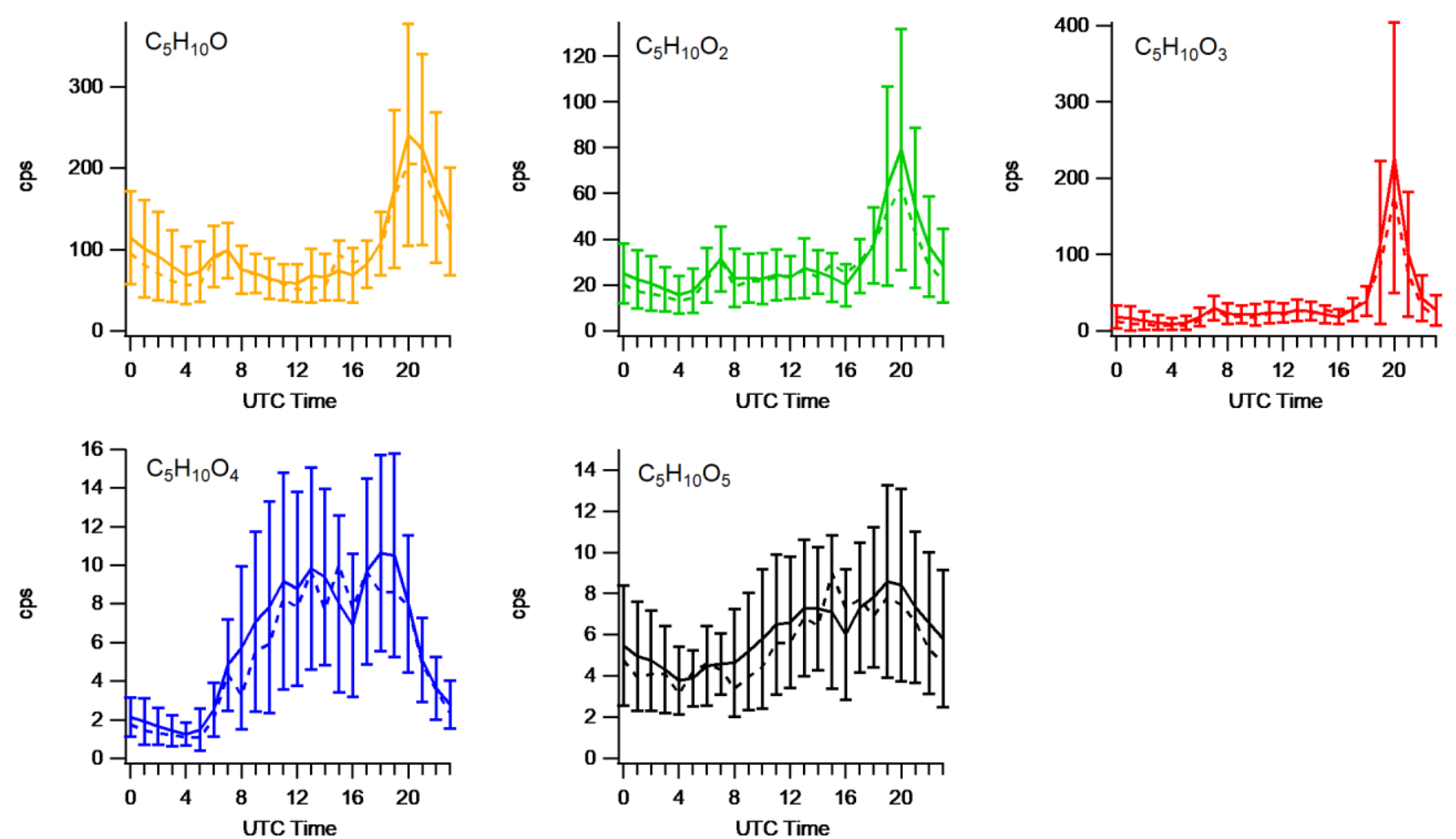

Figure S5. Diurnal patterns of non-nitrate isoprene oxidation products: (a) $\mathrm{C}_{5} \mathrm{H}_{10} \mathrm{O}$, (b) $\mathrm{C}_{5} \mathrm{H}_{10} \mathrm{O}_{2}$, (c) $\mathrm{C}_{5} \mathrm{H}_{10} \mathrm{O}_{3}$, (d) $\mathrm{C}_{5} \mathrm{H}_{10} \mathrm{O}_{4}$, and (e) $\mathrm{C}_{5} \mathrm{H}_{10} \mathrm{O}_{5}$. 

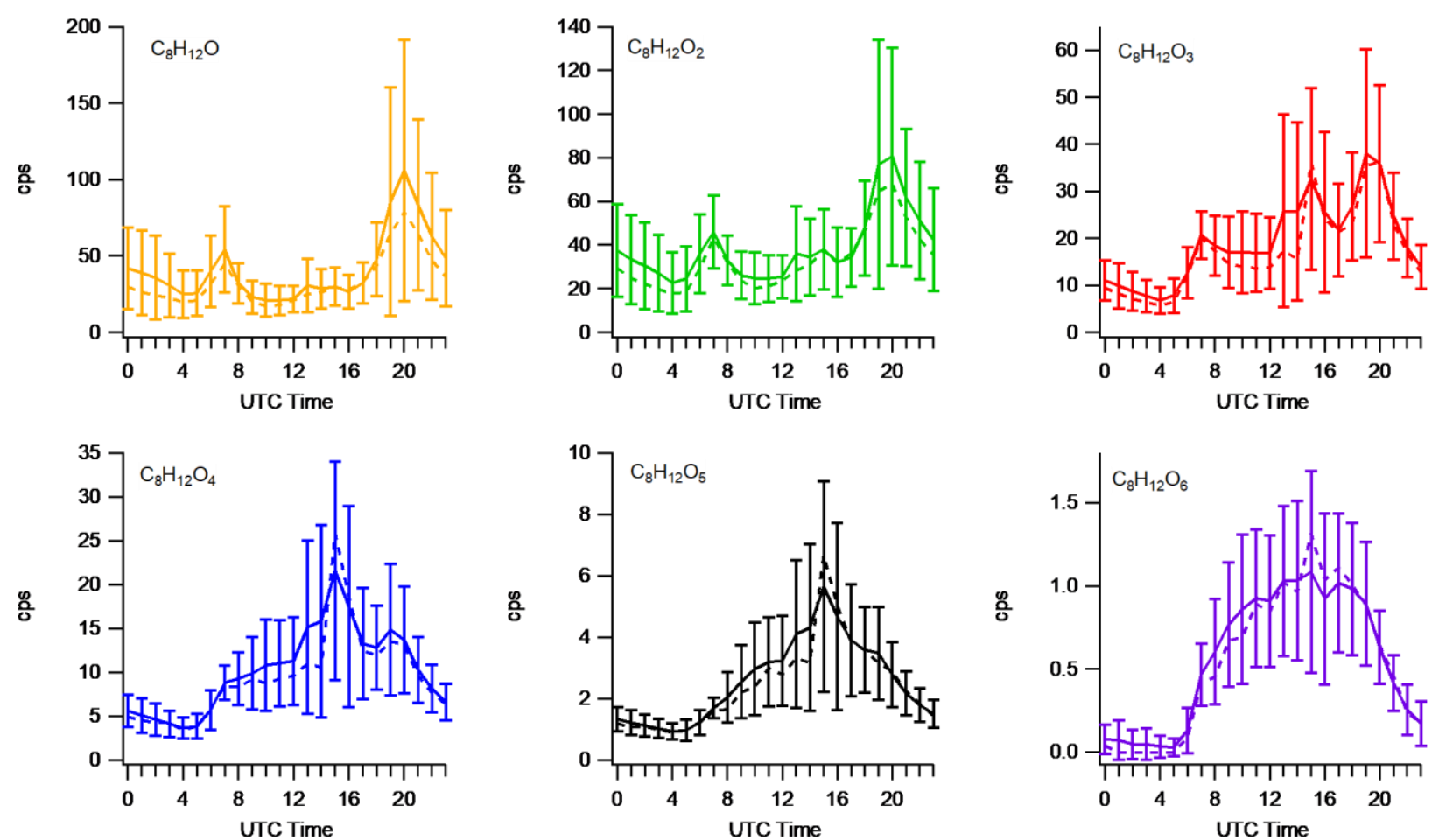

Figure S6. Diurnal patterns of non-nitrate monoterpene oxidation products: (a) $\mathrm{C}_{8} \mathrm{H}_{12} \mathrm{O}$, (b) $\mathrm{C}_{8} \mathrm{H}_{12} \mathrm{O}_{2}$, (c) $\mathrm{C}_{8} \mathrm{H}_{12} \mathrm{O}_{3}$, (d) $\mathrm{C}_{8} \mathrm{H}_{12} \mathrm{O}_{4}$, (e) $\mathrm{C}_{8} \mathrm{H}_{12} \mathrm{O}_{5}$, and (f) $\mathrm{C}_{8} \mathrm{H}_{12} \mathrm{O}_{6}$. 

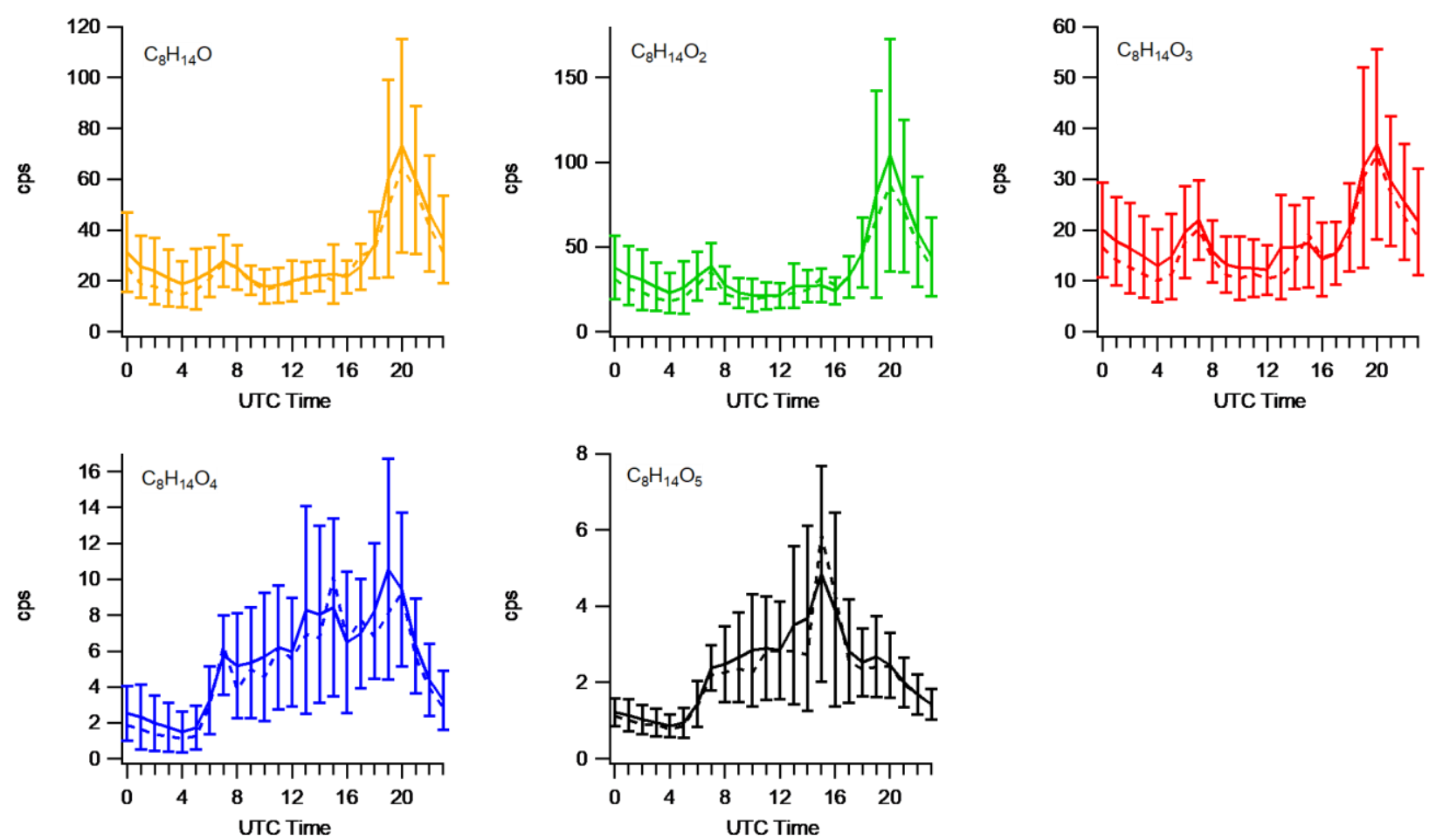

Figure S7. Diurnal patterns of non-nitrate monoterpene oxidation products: (a) $\mathrm{C}_{8} \mathrm{H}_{14} \mathrm{O}$, (b) $\mathrm{C}_{8} \mathrm{H}_{14} \mathrm{O}_{2}$, (c) $\mathrm{C}_{8} \mathrm{H}_{14} \mathrm{O}_{3}$, (d) $\mathrm{C}_{8} \mathrm{H}_{14} \mathrm{O}_{4}$, and (e) $\mathrm{C}_{8} \mathrm{H}_{14} \mathrm{O}_{5}$. 

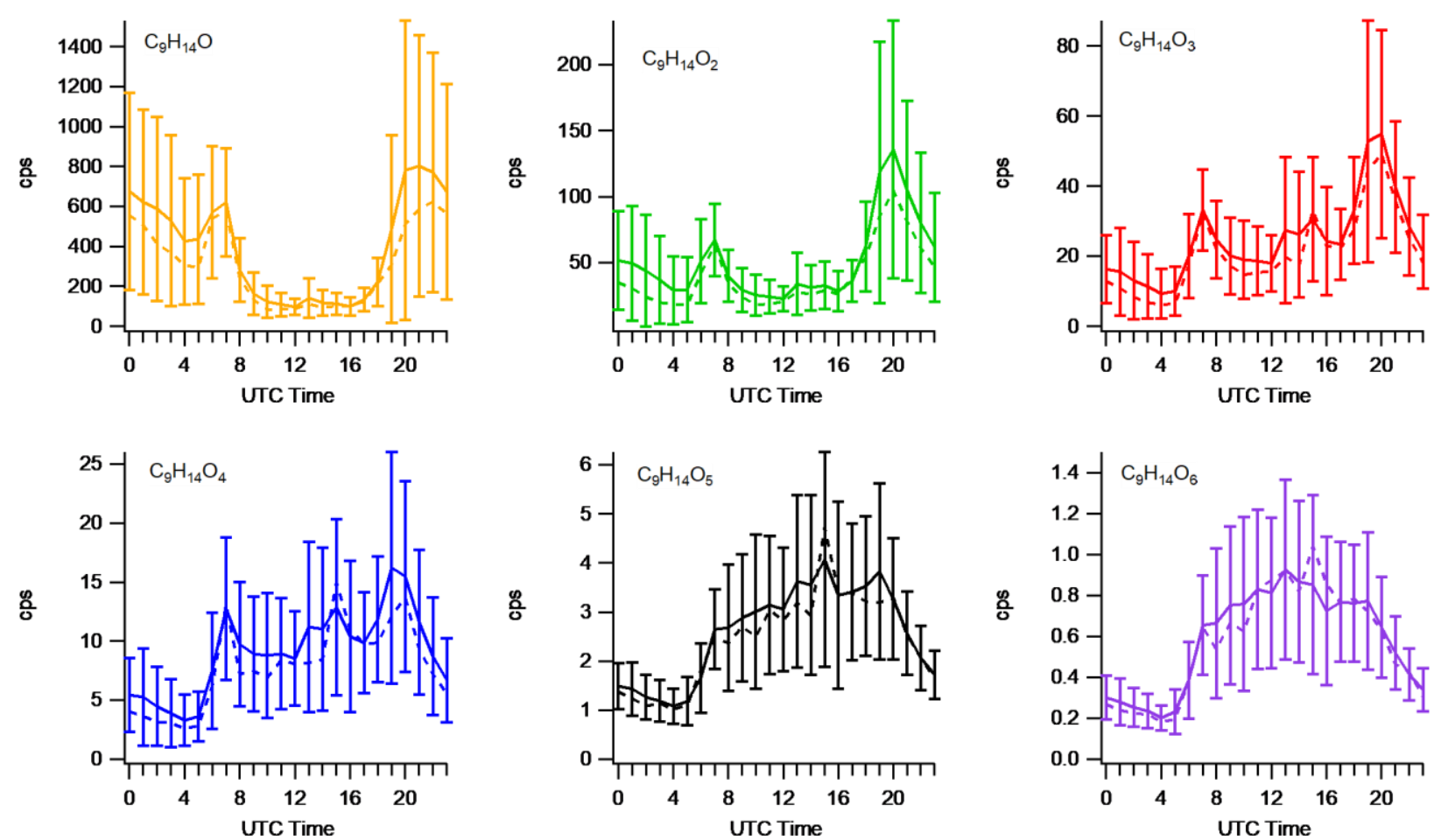

Figure S8. Diurnal patterns of non-nitrate monoterpene oxidation products: (a) $\mathrm{C}_{9} \mathrm{H}_{14} \mathrm{O}$, (b) $\mathrm{C}_{9} \mathrm{H}_{14} \mathrm{O}_{2}$, (c) $\mathrm{C}_{9} \mathrm{H}_{14} \mathrm{O}_{3}$, (d) $\mathrm{C}_{9} \mathrm{H}_{14} \mathrm{O}_{4}$, (e) $\mathrm{C}_{9} \mathrm{H}_{14} \mathrm{O}_{5}$, and (f) $\mathrm{C}_{9} \mathrm{H}_{14} \mathrm{O}_{6}$. 

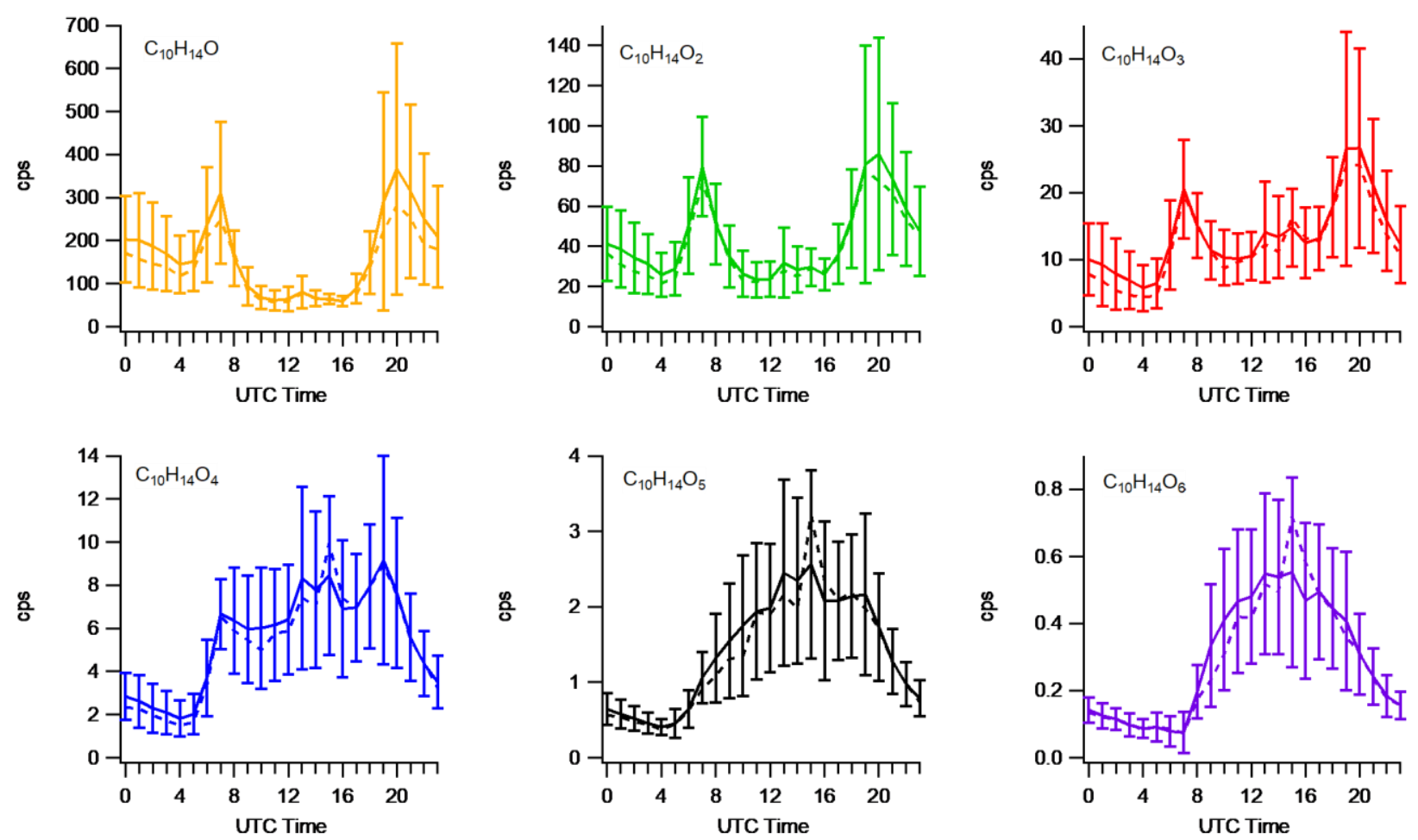

Figure S9. Diurnal patterns of non-nitrate monoterpene oxidation products: (a) $\mathrm{C}_{10} \mathrm{H}_{14} \mathrm{O}$, (b) $\mathrm{C}_{10} \mathrm{H}_{14} \mathrm{O}_{2}$, (c) $\mathrm{C}_{10} \mathrm{H}_{14} \mathrm{O}_{3}$, (d) $\mathrm{C}_{10} \mathrm{H}_{14} \mathrm{O}_{4}$, (e) $\mathrm{C}_{10} \mathrm{H}_{14} \mathrm{O}_{5}$, and (f) $\mathrm{C}_{10} \mathrm{H}_{14} \mathrm{O}_{6}$. 

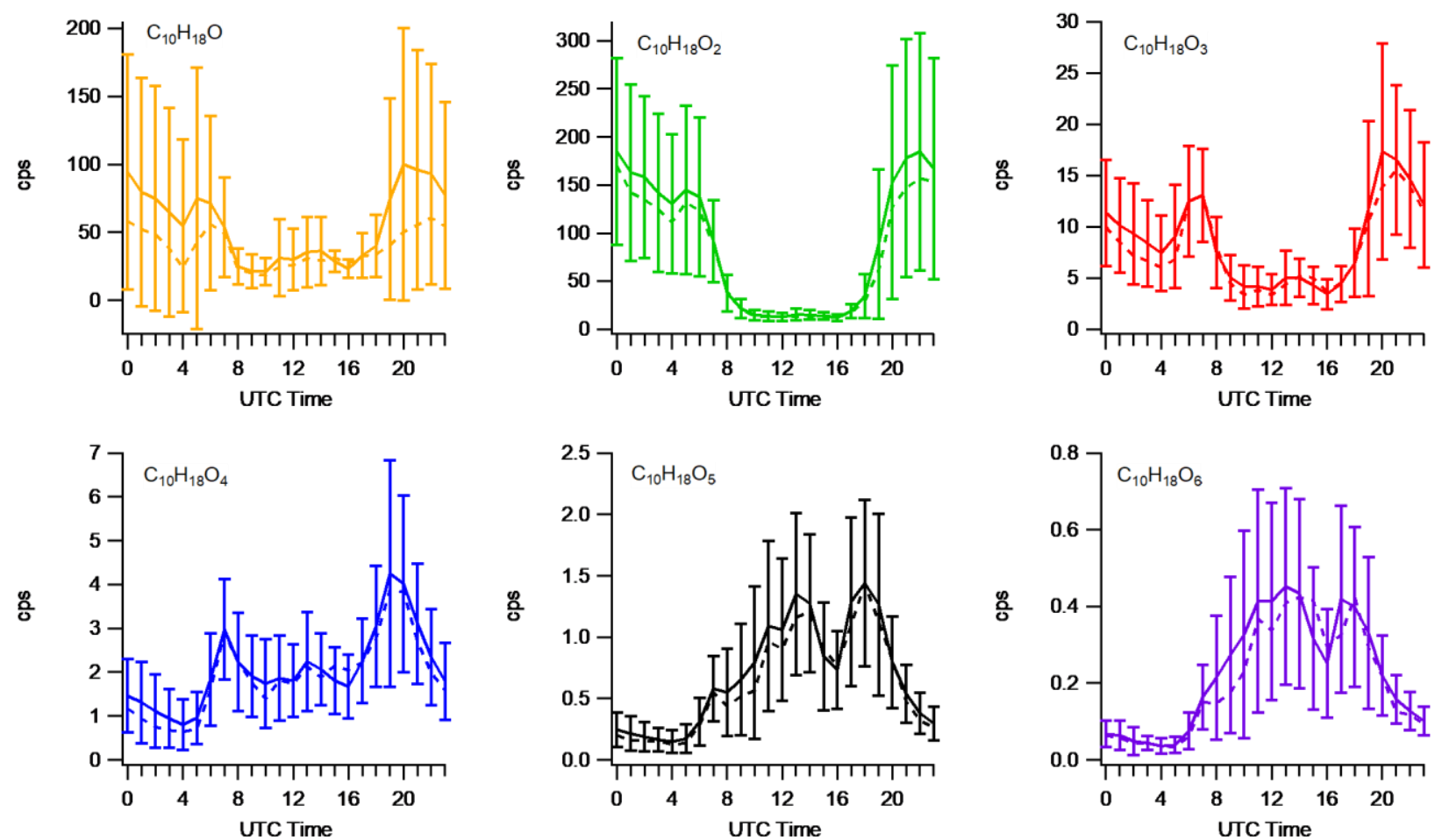

Figure S10. Diurnal patterns of non-nitrate monoterpene oxidation products: (a) $\mathrm{C}_{10} \mathrm{H}_{18} \mathrm{O}$, (b) $\mathrm{C}_{10} \mathrm{H}_{18} \mathrm{O}_{2}$, (c) $\mathrm{C}_{10} \mathrm{H}_{18} \mathrm{O}_{3}$, (d) $\mathrm{C}_{10} \mathrm{H}_{18} \mathrm{O}_{4}$, (e) $\mathrm{C}_{10} \mathrm{H}_{18} \mathrm{O}_{5}$, and (f) $\mathrm{C}_{10} \mathrm{H}_{18} \mathrm{O}_{6}$. 

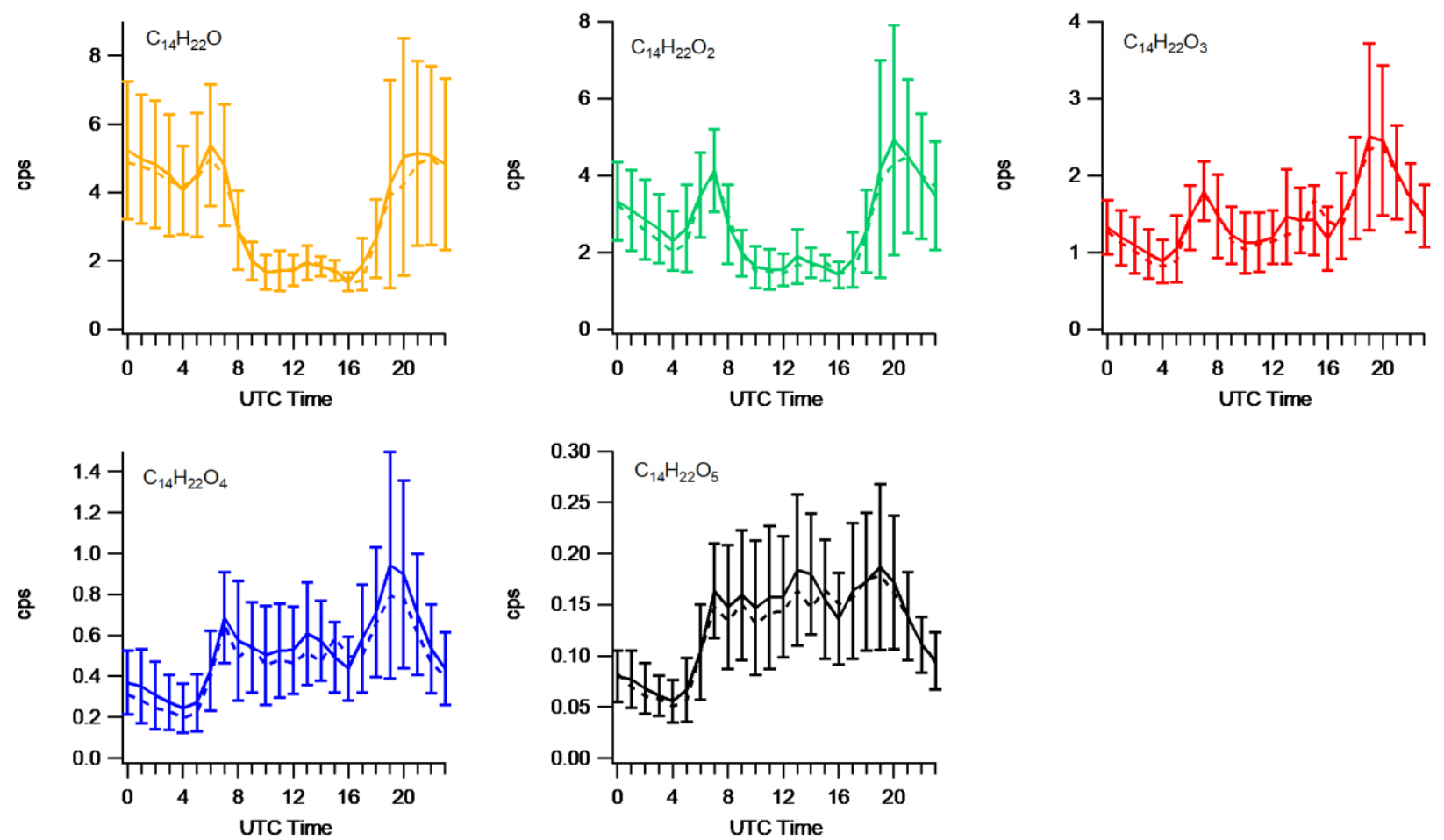

Figure S11. Diurnal patterns of non-nitrate sesquiterpene oxidation products: (a) $\mathrm{C}_{14} \mathrm{H}_{22} \mathrm{O}$, (b) $\mathrm{C}_{14} \mathrm{H}_{22} \mathrm{O}_{2}$, (c) $\mathrm{C}_{14} \mathrm{H}_{22} \mathrm{O}_{3}$, (d) $\mathrm{C}_{14} \mathrm{H}_{22} \mathrm{O}_{4}$, and (e) $\mathrm{C}_{14} \mathrm{H}_{22} \mathrm{O}_{5}$. 

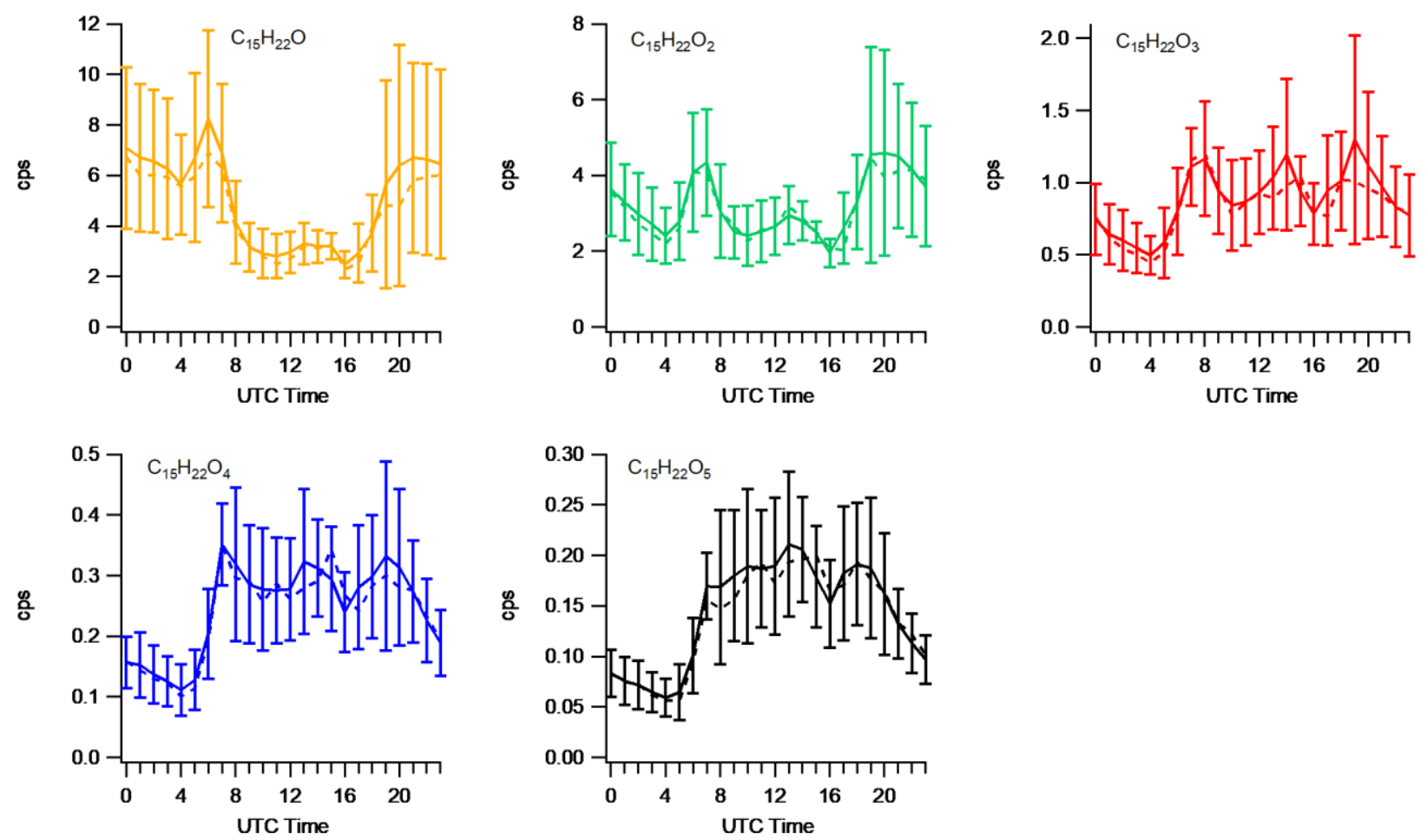

Figure S12. Diurnal patterns of non-nitrate sesquiterpene oxidation products: (a) $\mathrm{C}_{15} \mathrm{H}_{22} \mathrm{O}$, (b) $\mathrm{C}_{15} \mathrm{H}_{22} \mathrm{O}_{2}$, (c) $\mathrm{C}_{15} \mathrm{H}_{22} \mathrm{O}_{3}$, (d) $\mathrm{C}_{15} \mathrm{H}_{22} \mathrm{O}_{4}$, and (e) $\mathrm{C}_{15} \mathrm{H}_{22} \mathrm{O}_{5}$. 

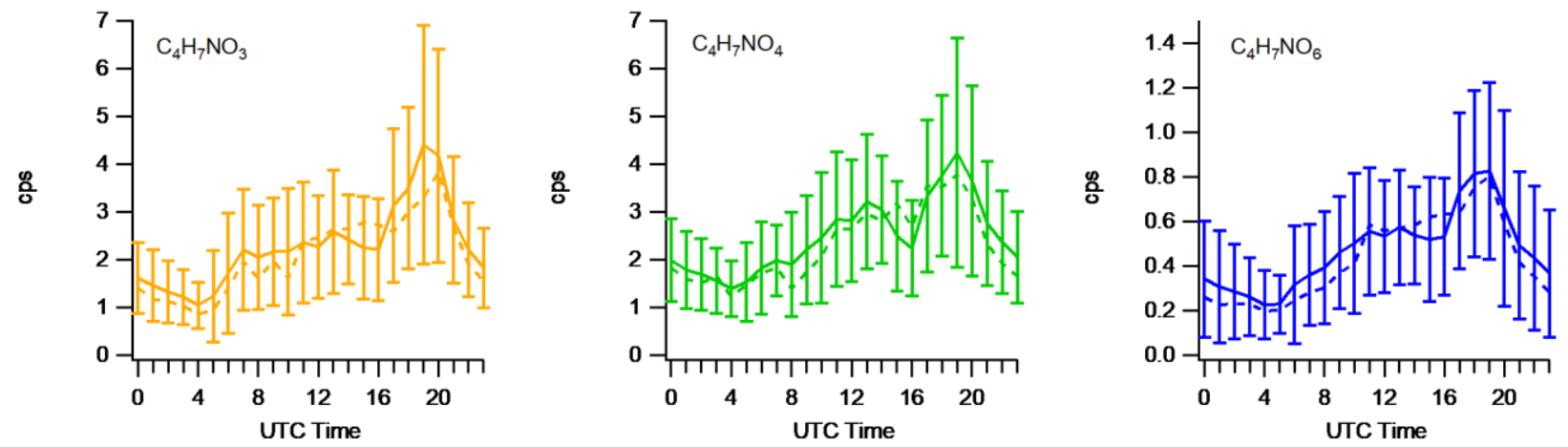

Figure S13. Diurnal patterns of isoprene-derived organic nitrates: (a) $\mathrm{C}_{4} \mathrm{H}_{7} \mathrm{NO}_{3}$, (b) $\mathrm{C}_{4} \mathrm{H}_{7} \mathrm{NO}_{4}$, and (c) $\mathrm{C}_{4} \mathrm{H}_{7} \mathrm{NO}_{6}$. 

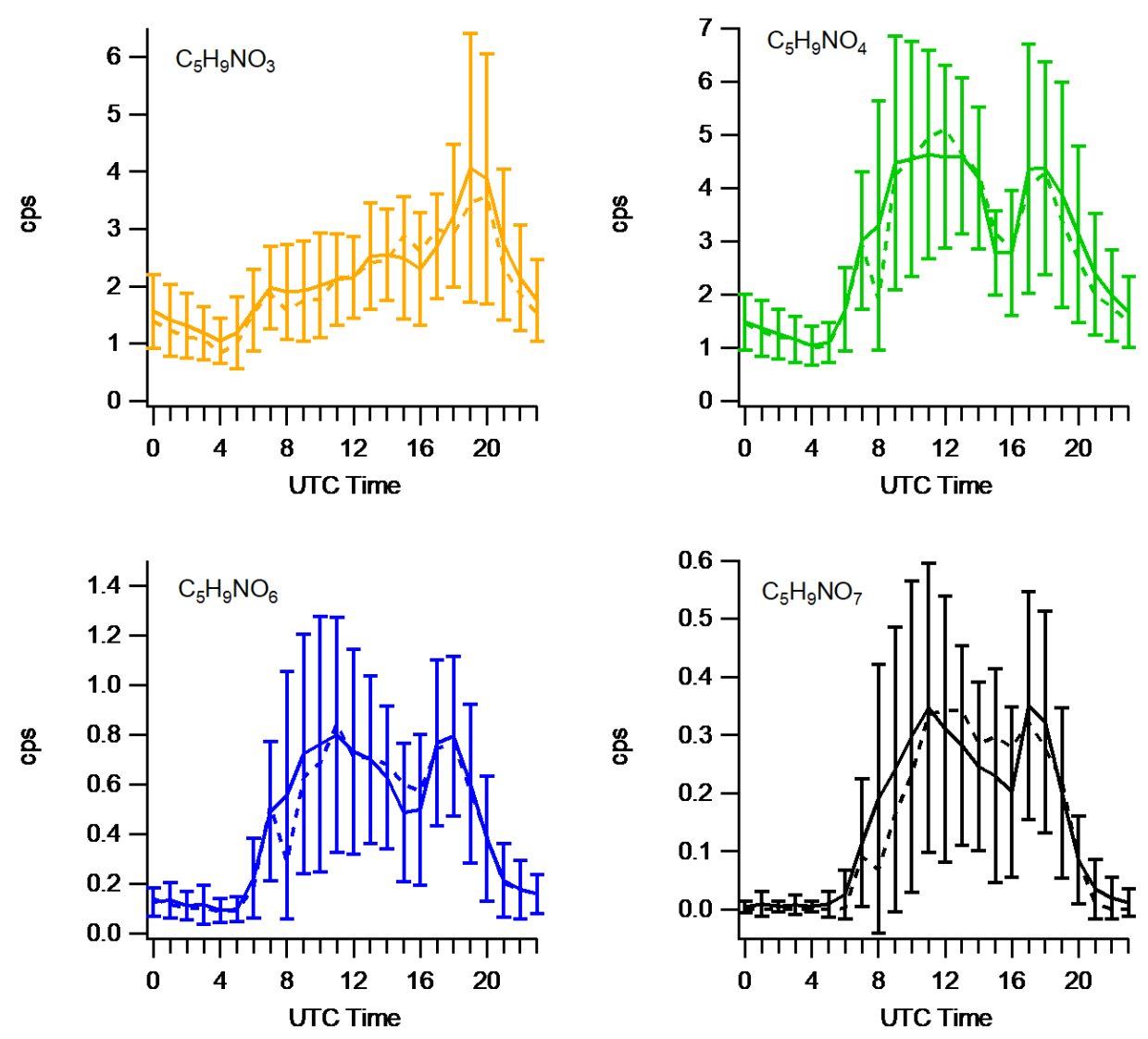

Figure S14. Diurnal patterns of isoprene-derived organic nitrates: (a) $\mathrm{C}_{5} \mathrm{H}_{9} \mathrm{NO}_{3}$, (b) $\mathrm{C}_{5} \mathrm{H}_{9} \mathrm{NO}_{4}$, (c) $\mathrm{C}_{5} \mathrm{H}_{9} \mathrm{NO}_{6}$, and (d) $\mathrm{C}_{5} \mathrm{H}_{9} \mathrm{NO}_{7}$. 

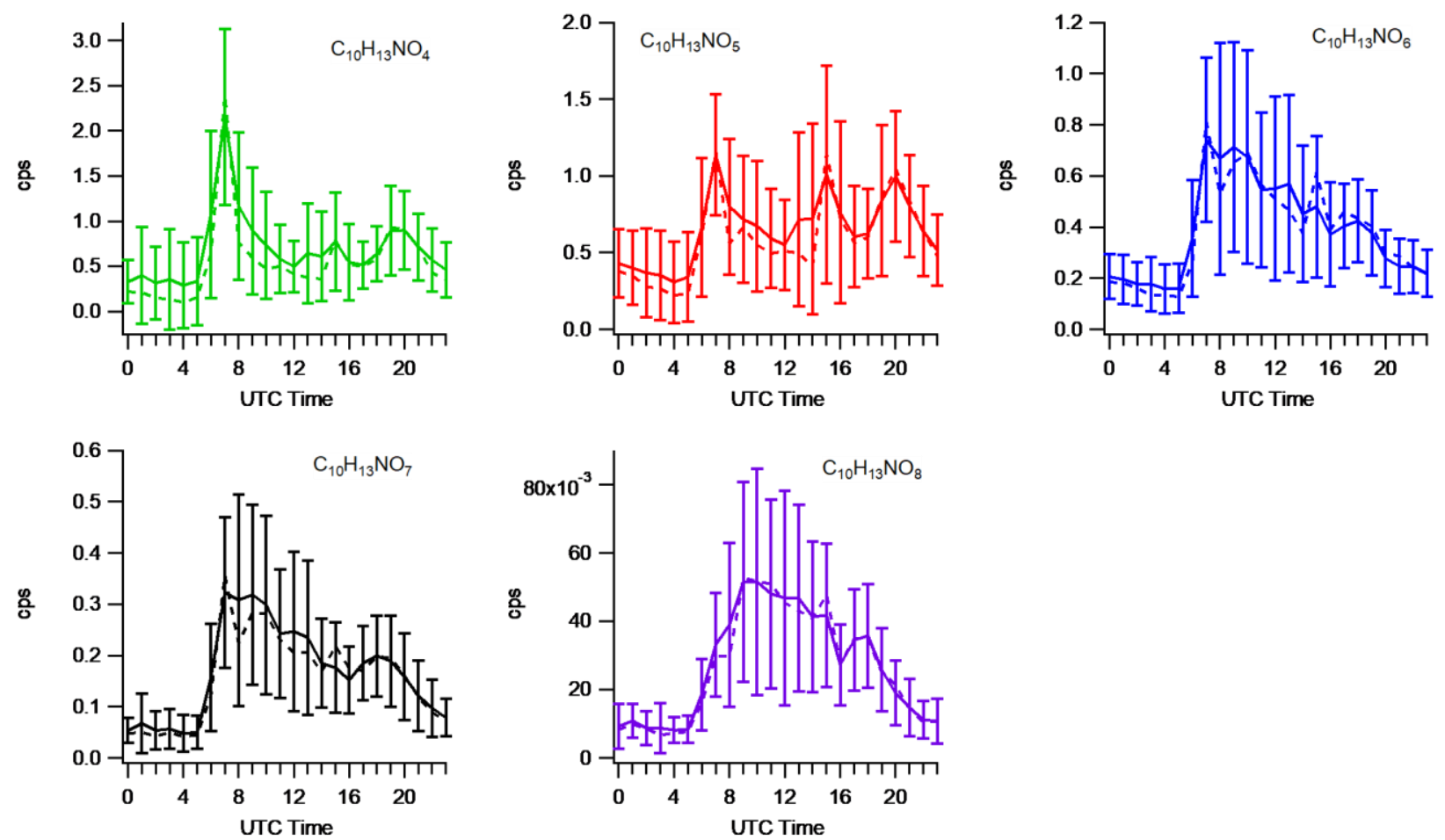

Figure S15. Diurnal patterns of monoterpene-derived organic nitrates: (a) $\mathrm{C}_{10} \mathrm{H}_{13} \mathrm{NO}_{4}$, (b) $\mathrm{C}_{10} \mathrm{H}_{13} \mathrm{NO}_{5}$, (c) $\mathrm{C}_{10} \mathrm{H}_{13} \mathrm{NO}_{6}$, (d) $\mathrm{C}_{10} \mathrm{H}_{13} \mathrm{NO}_{7}$, and (e) $\mathrm{C}_{10} \mathrm{H}_{13} \mathrm{NO}_{8}$. 

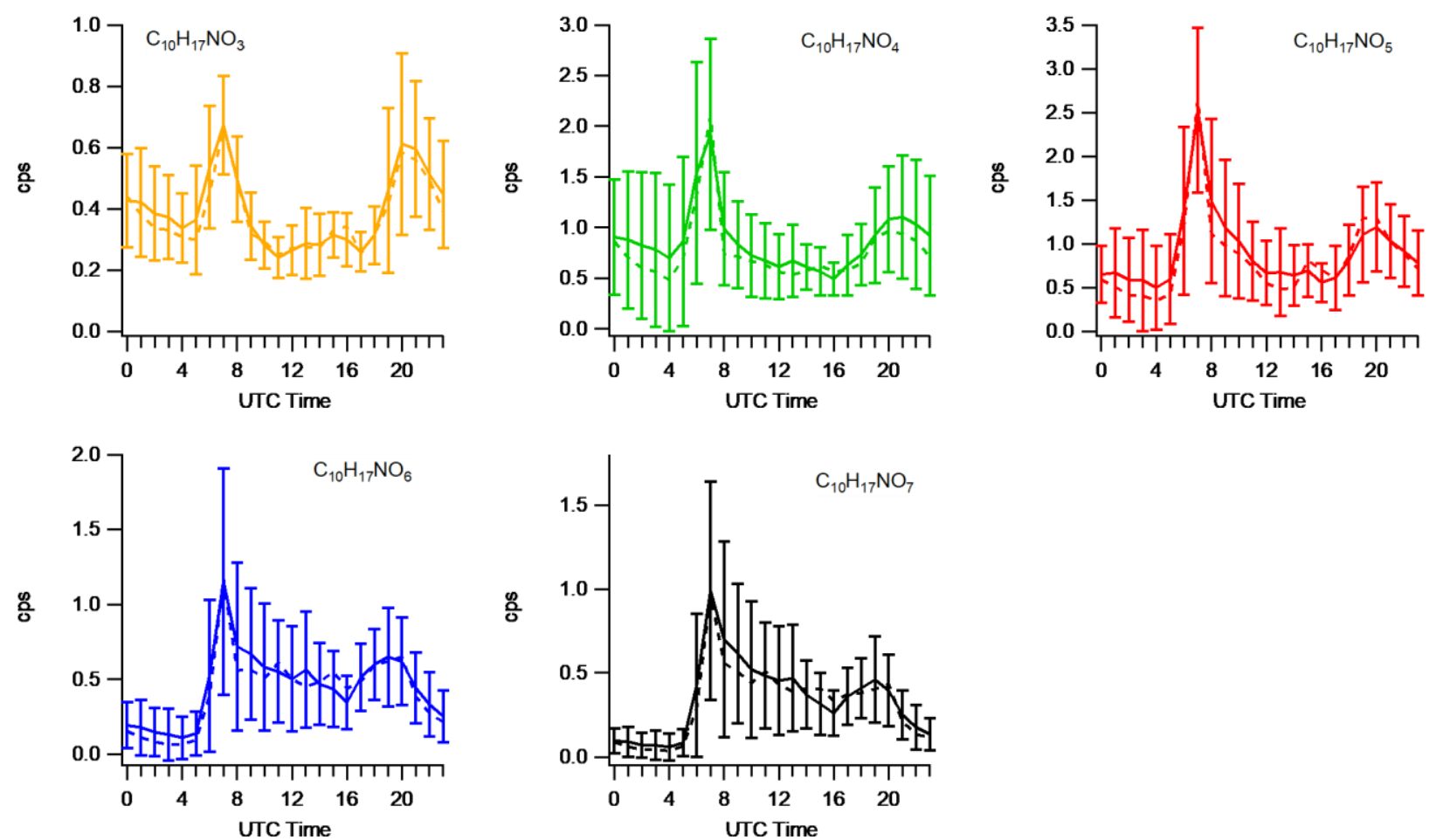

Figure S16. Diurnal patterns of monoterpene-derived organic nitrates: (a) $\mathrm{C}_{10} \mathrm{H}_{17} \mathrm{NO}_{3}$, (b) $\mathrm{C}_{10} \mathrm{H}_{17} \mathrm{NO}_{4}$, (c) $\mathrm{C}_{10} \mathrm{H}_{17} \mathrm{NO}_{5}$, (d) $\mathrm{C}_{10} \mathrm{H}_{17} \mathrm{NO}_{6}$, and (e) $\mathrm{C}_{10} \mathrm{H}_{17} \mathrm{NO}_{7}$. 